\title{
LETTERS
}

\section{High mortality is expected in patients who have COVID-19 during the postoperative period}

Since coronavirus disease 2019 (COVID-19) was declared a pandemic by the World Health Organization on Mar. 11, 2020, many professional societies have recommended that elective surgeries be postponed if possible, with the goal of minimizing disease spread and maintaining health care capacity. Even so, the provision of surgery will continue to be an essential part of our health care system throughout the pandemic. ${ }^{1}$

For patients who need surgery, what is their risk if they are infected with severe acute respiratory syndrome coronavirus 2 (SARS-CoV-2) postoperatively? So far, little is known about the impact of COVID-19 on mortality in patients during the postoperative period. I searched PubMed for articles published up to May 13, 2020, using the search terms "novel coronavirus," "2019-nCoV," "COVID-19" or "SARS-CoV-2" with no language or time restriction. I found 3 articles including 19 patients who had COVID-19 perioperatively. ${ }^{2-4}$ Of the 19 patients, 11 were male (57.9\%), and the mean age was 60.3 years. The operations included general, gynecologic and thoracic surgeries. Information about the timing of COVID-19 symptom onset was available in 12 patients; 9 patients (75\%) had pneumonia symptoms within 7 days after surgery and the other 3 had symptoms more than 7 days after surgery. The case fatality rate was $36.8 \%(7 / 19)$.

These data suggest that patients infected with SARS-CoV-2 in the perioperative period have a high risk of death, although there may be a bias to report more severe cases. It is widely believed that the fatality of COVID-19 in the general population is less than $5 \%$, but older age and comorbidities are indicators of poor prognosis. ${ }^{5}$ Data here suggest that postoperative patients may be another group who have a poor prognosis with COVID-19.

The median incubation period of SARSCoV-2 has been reported to be 4 days, with a range of about 2-7 days. ${ }^{6}$ The fact that pneumonia symptoms developed in $75 \%$ of the patients within 7 days after surgery means that most of the infections may have occurred before surgery. These surgeries could have been delayed safely if SARS-CoV-2 infection had been detected before the operations. Because no vaccine or effective antiviral treatments are available, prevention is the best way to reduce the impact of COVID-19. Preoperative intensive surveillance for SARS-CoV-2 infection must be done during the pandemic. To avoid nosocomial transmission of the virus, strict isolation and protection measures should be implemented. Visitor policies should be updated simultaneously; each patient should have only 1 visitor at a time. Finally, our surgical communities should adapt to the unprecedented worldwide public health crisis to develop a COVID-19-specific approach to providing the very best care to our patients during the pandemic.

\section{Gengwen Huang MD PhD}

General surgeon, Department of General Surgery, Xiangya Hospital, Central South University, Changsha, Hunan, China

Cite as: CMAJ 2020 July 20;192:E847. doi: $10.1503 / \mathrm{cmaj} .75781$

\section{References}

1. Urbach DR, Martin D. Confronting the COVID-19 surgery crisis: time for transformational change. CMAJ 2020;192:E585-6.

2. Aminian A, Safari S, Razeghian-Jahromi A, et al. COVID-19 outbreak and surgical practice: unexpected fatality in perioperative period. Ann Surg 2020;272:e27-9.

3. Yang S, Zhang Y, Cai J, et al. Clinical characteristics of COVID-19 after gynecologic oncology surgery in three women: a retrospective review of medical records. Oncologist 2020;25:e982-5.

4. Li YK, Peng S, Li LQ, et al. Clinical and transmission characteristics of COVID-19: a retrospective study of 25 cases from a single thoracic surgery department. Curr Med Sci 2020;40:295-300.

5. Zhou F, Yu T, Du R, et al. Clinical course and risk factors for mortality of adult inpatients with COVID-19 in Wuhan, China: a retrospective cohort study. Lancet 2020;395:1054-62.

6. Guan W, Ni Z, Hu Y, et al. Clinical characteristics of coronavirus disease 2019 in China. N Engl J Med 2020;382:1708-20.

Competing interests: None declared. 